\title{
A lattice discrete element method to model the falling-weight impact test of PMMA specimens
}

\author{
Luis E. Kosteski ${ }^{\mathrm{a}, *}$, Ignacio Iturrioz ${ }^{\mathrm{b}}$, Adrián P. Cisilino ${ }^{\mathrm{c}}$, Ricardo Barrios D’ambra ${ }^{\mathrm{d}}$, \\ Valeria Pettarin ${ }^{c}$, Laura Fasce ${ }^{c}$, Patricia Frontini ${ }^{\mathrm{c}}$ \\ a PPENG, Engenharia Mecânica, Universidade Federal do Pampa - Campus Alegrete, Av. Tiarajú, 810, Bairro: Ibirapuitã, Alegrete, RS, Brazil \\ b PROMEC, Universidade Federal do Rio Grande do Sul, Sarmento Leite 425, Porto Alegre, RS, Brazil \\ c INTEMA, Facultad de Ingeniería, Universidad Nacional de Mar del Plata, Av. Juan B. Justo 4302, Mar del Plata, Buenos Aires, Argentina \\ ${ }^{d}$ Departamento de Mecánica Aplicada, Facultad de Ingeniería, Universidad Nacional del Nordeste, Av. Las Heras 727, Resistencia, Chaco, Argentina
}

\section{A R T I C L E I N F O}

\section{Article history:}

Available online 23 June 2015

\section{Keywords:}

Impact test

Impact of polymers

Lattice discrete element methods

Abaqus

Explicit

\begin{abstract}
A B S T R A C T
It is introduced in this paper a Lattice Discrete Element Method (LDEM) for modelling the fallingweight test of polymethyl-methacrylate (PMMA) specimens. The method exploits the inherent characteristics of discrete methods to model crack initiation and propagation by simply breaking the links between their discrete components. It results in a flexible modelling tool that is implemented using Abaqus/Explicit. Numerical results are validated by comparison with experimental tests. The results are compared in terms of the time evolution of the striker force and velocity and the specimen crack patterns. The LDEM simulations are, in every case, of predictive nature. Material properties are neither left open for calibration nor used to adjust the numerical results. There is a good agreement between experimental and numerical results. It is shown that the proposed LDEM has the capability to capture all the main features of the sequence of events that occur during the experiment: the elastic specimen loading prior to the crack initiation, the nucleation and propagation of radial cracks as the test progresses, and the final failure after the rapid propagation of a circular crack that joins the radial cracks together. The effects of the variability of the material fracture toughness on the test results are studied using a series of models with random distribution of the fracture energy.
\end{abstract}

(c) 2015 Elsevier Ltd. All rights reserved.

\section{Introduction}

The utilization of polymers and their composites in engineering applications demands the constant improvement of test methods and analysis tools to better understand their failure mechanics failure. One standard test for the impact characterization of polymers is the falling-weight test. In this test, a disk-shaped specimen is subjected to the impact of a steel striker with a hemispherical head to induce a biaxial stress state. The impact energy is controlled by means of the striker mass and velocity [15].

The finite element method is well developed for the simulation of dynamic crack propagation. The two main approaches for this task are cohesive interface elements and the extended finite element method (XFEM). Cohesive elements were initially proposed by Xu and Needleman [37] and further developed by Pandolfi and Ortiz [25], among others. Cohesive elements are inserted adaptively on the element interfaces when the effective traction acting

\footnotetext{
* Corresponding author.

E-mail address: luiskosteski@hotmail.com (L.E. Kosteski).
}

on those interfaces reaches the cohesive strength. Since the insertion of the cohesive elements is limited to the element interfaces, this approach might perform poorly for crack paths that are not coincident with meshlines or when the model discretization is not enough refined. For its part, the XFEM introduced by Belytschko et al. [4], injects the discontinuity into the element interpolation functions on the basis of a failure criterion and level sets. XFEM is not as sensitive as the cohesive element approach to model discretization, but the use of level sets to identify the failure zone tends to favour the propagation of a single crack, and thus the inhibition of crack branching. Song et al. [35] have also pointed out that cohesive elements perform better than XFEM to model crack nucleation.

Discrete methods are specially suited for the simulation of dynamic crack propagation problems. In contrast to the finite element method, discrete methods have the natural ability to introduce discontinuities in a very direct and intuitive way by simply breaking the links between their discrete components. Moreover, discrete methods offer a convenient framework to account for the disorder of the material microstructure by means of statistical models. This feature is an advantage over traditional continuum micromechanical models, which adopt homogenization tech- 
niques to convert a disordered material into an equivalent continuum model. Different methods such as discrete elements, discontinuous deformation analysis and molecular dynamics can be formulated depending on the individual element introduced: particles, agents or molecules.

In the classical discrete element method (DEM), the material is represented as a collection of spherical (in 3D) or cylindrical (in 2D) discrete elements interacting among themselves via contact forces. Contact interaction models employ the decomposition of the contact force into normal and tangential components. The translational and rotational motions of the elements (particles) are governed by the standard equations of rigid body dynamics [5]. As in DEM, peridynamic methods represent the material by a collection of particles, but they interact directly and non-locally with all particles that lie within a given distance [34]. Peridynamic theory offers a mathematically consistent technique for modelling solid bodies with continuous and discontinuous displacements as well as a method that unifies the mechanics of particles and continuum bodies through the utilization of long-range forces.

Lattice discrete element methods (LDEM) represent the continuum as a periodic spatial arrangement of bars with masses lumped at their ends. Lattice methods have been successfully employed in a number of applications, like to solve small deformations of generally anisotropic plane continua [8], to model crack propagation [31] and to estimate the damage and effective mechanical properties of disordered microstructures [26,27]. The LDEM used in this work can be assimilated to a reverse homogenization method [33]. Nayfeh and Hefzy [24] developed and approach to model a large panel made of interconnected bars as an equivalent orthotropic elastic continuum. Conversely, the LDEM represents an orthotropic continuum by an equivalent regular truss lattice. Rocha et al. [30], and more recently Kosteski et al. [16], extended the LDEM to account for to the irreversible effects of crack nucleation and propagation. Since then, the method have found applications in the analysis of impulsive loads in shells [28], fracture of elastic foundations on soft sand beds [32], scale effects in concrete [29] and rocks dowels $[13,21]$ and fracture and damage propagation $[16,18,22]$.

It is introduced in this work an LDEM for the simulation of the falling-weight impact response of polymethyl-methacrylate (PMMA) specimens. With the aim to develop a flexible modelling tool, the LDEM is implemented using Abaqus/Explicit [7]. This allows combining the LDEM to the Abaqus capabilities for contact and eigenvalue analyses, and for the inclusion of rigid parts into the model assembly. Numerical analyses are complemented with experimental tests for the material characterization and the model validation. A series of LDEM simulations are conducted in order to account for the variability in the material properties. Numerical and experimental results are compared in terms of the time evolution of the striker force and velocity, and the specimen crack pattern. The time evolutions of the kinetic, elastic and fracture energies are analyzed, and their behaviours are correlated to the sequence of crack initiation, nucleation and propagation events that occur during the test.

\section{The lattice discrete element method}

The lattice discrete element method (LDEM) used in this work represents the continuum by means of a periodic spatial arrangement of bars with masses lumped at their ends. The discretization strategy is due to Nayfeh and Hefzy [24], and it is shown in Fig. 1(a) and (b). The discretization uses a basic cubic module constructed using 20 bar elements and nine nodes. Every node has three degrees of freedom, which are the three components of the displacement vector in the global reference system.

The lumped masses at the nodes are specified in such a way that they add up the mass of the continuum the module represents, $m_{\text {module }}=\rho \cdot L^{3}$. Thereby, one half the module mass is assigned to the node at the module centre, $m_{\text {centre }}=0.5 \rho L^{3}$, while the other half of the mass is distributed evenly among the eight corner nodes. It is worth noting that, since neighbour modules share their corner nodes, these nodes account for the contributions of multiple modules after the complete model assembly.

In the case of an isotropic elastic material, the equivalent axial stiffness per unit length of the longitudinal elements (those located along module edges and those connecting the nodes in the centre of the modules) is:

$E_{l}^{A}=A_{l} E=\phi E L^{2}$,

where $A_{l}$ is the cross-sectional area of the element and $E$ is the Young's modulus of the solid being discretized. The function $\phi=(9+8 \delta) /(18+24 \delta)$, where $\delta=9 v /(4-8 v)$, accounts for the effect of the Poisson's ratio $v[6,24]$. Similarly, the stiffness of the diagonal elements is

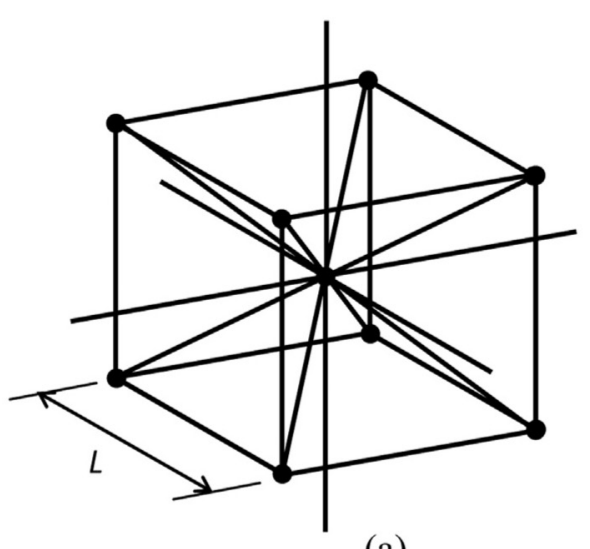

(a)

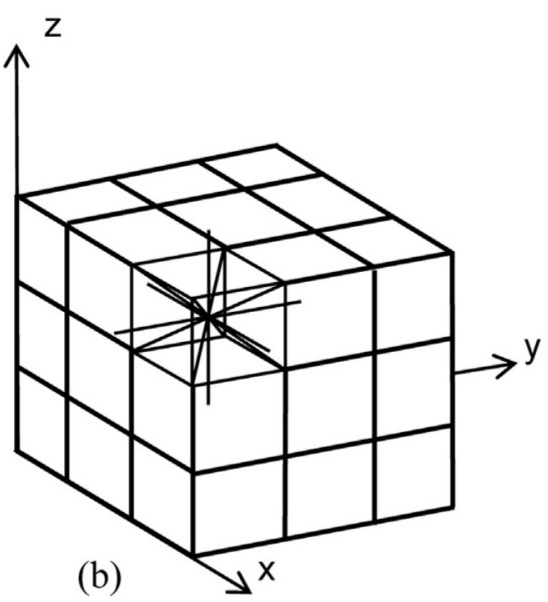

Fig. 1. Discretization strategy of the LDEM: (a) basic cubic module, (b) prism formed using several cubic modules. 
$E_{d}^{A}=A_{d} E=\frac{2}{\sqrt{3}} \delta \phi E L^{2}$.

The coefficient $2 / \sqrt{3}$ in equation (2) accounts for the difference in length between longitudinal and diagonal elements, this is, $L=2 / \sqrt{3} L_{d}$.

It is important to point out that for $v=0.25$, the correspondence between the equivalent discrete solid and the isotropic continuum is complete. On the other hand, discrepancies appear in the shear terms for values of $v \neq 0.25$. These discrepancies are small and may be neglected in the range $0.20 \leq v \leq 0.30$. Different element arrays can be adopted for materials with $v$ outside this range (see Ref. [24]). It is interesting to note that while no lattice model can exactly represent a locally isotropic continuum, it can also be argued that no perfect locally isotropic continuum exists in practical engineering applications. Isotropy in solids is a bulk property that reflects the random distribution of the constituent elements orientation. A comprehensive study on the effect of the LDEM lattice geometry on the Poisson's ratio can be found in Ref. [27].

Newton's second law is enforced at every node to obtain the system of equations

$\mathbf{M} \ddot{\mathbf{x}}+\mathbf{C} \dot{\mathbf{x}}+\mathbf{F}(t)-\mathbf{P}(t)=0$

where vectors $\ddot{\mathbf{x}}$ and $\dot{\mathbf{x}}$ contain the nodal accelerations and velocities; $\mathbf{M}$ and $\mathbf{C}$ are the mass and damping matrices respectively, and the vectors $\mathbf{F}(t)$ and $\mathbf{P}(t)$ contain the nodal internal and external forces. Since $\mathbf{M}$ and $\mathbf{C}$ are diagonal, the equations in expression (3) are not coupled and they can be easily integrated in the time domain using an explicit finite difference scheme.

Since nodal coordinates are updated at every time step, the LDEM naturally accounts for large displacements. At the same time, the LDEM has a natural ability to model cracks. Cracks can be introduced into the models either as pre-existent features, or they can result from the process of crack nucleation and propagation. Crack nucleation and propagation require of non-linear constitutive models that allow the elements to break when they attain a critical condition. The details about the formulation and implementation of these non-linear constitutive models are given in the next section.

\subsection{Non-linear constitutive model for material damage}

Rocha et al. [30], and more recently Kosteski et al. [16], introduced non-linear constitutive models to account for the reduction in the element load carrying capacity due to crack nucleation and propagation. The bilinear model for quasi-brittle materials due to Rocha et al. [30] is used in this work and it will be briefly presented next. The readers are referred to the above references for further details.

The non-linear constitutive model is in Fig. 2. The area under the force vs. strain plot (the area of the triangle $O A B$ ) is the energy density necessary to fracture the area of influence of the element. Thus, for a given point $P$ on the force vs. strain curve, the area of the triangle $O P C$ represents the reversible elastic energy density stored in the element, while the area of the triangle OAP is the dissipated fracture energy density. Once the dissipated energy equals the fracture energy, the element fails and loses its load carrying capacity. On the other hand, in the case of compressive loads the material behaves as linear elastic. Thus, the failure in compression is induced by indirect traction. This assumption is reasonable for quasi-brittle materials for which the ultimate strength in compression is usually from five to ten times larger than that in tension (see Ref. [19]).

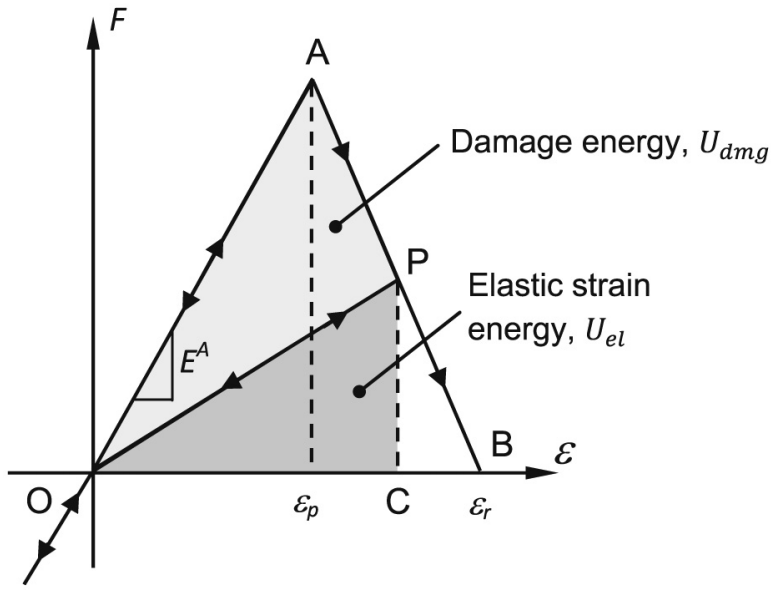

Fig. 2. Bilinear constitutive model with material damage.

The parameters for the constitutive model in Fig. 2 are (see Ref. [30]):

- Force, F: the element axial force as a function of the longitudinal strain $\varepsilon$.

- Element stiffness, $E_{i}^{A}$ : depending whether a longitudinal or a diagonal element is considered, $E_{d}{ }^{A}$ or $E_{l}^{A}$ is adopted, see equations (1) and (2).

- Length of the LDEM module, $L$.

- Specific fracture energy, $G_{f}$ : it is specified as the material fracture energy, $G_{c}$.

- Equivalent fracture area, $A_{l}^{f}$ : this parameter enforces the equivalence between the fracture energy dissipated by the continuum and by its discrete element representation. The energy dissipated by the fracture of a material sample of size $L \times L \times L$ due to a crack parallel to one of its faces is:

$$
\Gamma=G_{f} \Delta=G_{f} L^{2} .
$$

The energy dissipated by the fracture of an LDEM module is,

$$
\Gamma_{L D E M}=G_{f} \cdot c_{A}\left[4 \cdot 0.25+1+4\left(\frac{2}{\sqrt{3}} \delta\right)\right] L^{2},
$$

where the first term accounts for the four edge normal elements, the second term for the internal longitudinal element, and the third term for the four diagonal elements. The factor $(2 \delta / \sqrt{3})$ in the third term is the ratio between the diagonal and the longitudinal bar stiffnesses, which is the quotient between expressions (2) and (1). The coefficient $c_{A}$ is a scaling parameter that imposes the equivalence between $\Gamma$ and $\Gamma_{L D E M}$. Equating equations (4) and (5) for the case $v=0.25$, it results

$G_{f} L^{2} \cong G_{f}\left(\frac{22}{3} c_{A}\right) L^{2}$,

from which the value $c_{A} \cong 3 / 22$ is obtained.

Finally, the equivalent fracture areas of longitudinal and diagonal elements are:

$A_{d}^{f}=\frac{3}{22} L^{2}$

and 


$$
A_{l}^{f}=\frac{4}{22} L^{2}
$$

for the diagonal and longitudinal elements, respectively.

- Critical failure strain, $\varepsilon_{p}$ : the maximum strain attained by the elements before damage initiation (point A in Fig. 2). This is related to the specific fracture energy as

$$
\varepsilon_{p}=R_{f} \sqrt{\frac{G_{f}}{E}},
$$

where $R_{f}$ is the so-called failure factor, which accounts for the presence of an intrinsic crack of length $\ell$ by means of

$$
R_{f}=\frac{1}{Y \sqrt{\ell}}
$$

where $Y$ is a dimensionless parameter that depends on both, the specimen and the crack geometry. Note that the length of the intrinsic defect is predetermined, and it could be considered as a material property.

- Limit strain, $\varepsilon_{r}$ : strain value for which the elements lose their load carrying capacity (point B in Fig. 2). The limit strain is expressed in terms of the critical strain

$$
\varepsilon_{r}=K_{r} \varepsilon_{p}
$$

where

$$
K_{r}=\left(\frac{G_{f}}{E \varepsilon_{p}^{2}}\right)\left(\frac{A_{i}^{f}}{A_{i}}\right)\left(\frac{2}{L_{i}}\right)
$$

is set to satisfy the dissipated fracture energy balance.

The condition $K_{r} \geq 1$ must be fulfilled to guarantee the stability of the explicit formulation. This condition is enforced be means of the restriction $L_{i} \leq L_{c r}$ on the element length, where

$$
L_{c r}=2\left(\frac{G_{f}}{E \varepsilon_{p}^{2}}\right)\left(\frac{A_{i}^{f}}{A_{i}}\right)
$$

for both, the longitudinal, $i=l$, and the diagonal elements, $i=d$. The values for the element cross-sectional areas, $A_{i}$, are those given in expressions (1) and (2), while the equivalent fracture areas, $A_{i}^{f}$, are in equations (7) and (8).

It is interesting to note that in contrast to the usual practice in finite elements, the constitutive relationship in the LDEM is not only a function of the material properties. The element constitutive relationship introduced above is defined in terms of material properties, $\varepsilon_{p}, E, R_{f}$ and $G_{f}$; parameters that depend on model discretization, $A_{i}^{f}$ and $L$; and parameters that depend on both, material properties and model discretization, $E_{d}{ }^{A}$ and $\varepsilon_{r}$. A second interesting feature of the method is that, although it uses a scalar damage law to describe the uniaxial behaviour of the elements, the global model accounts for anisotropic damage, since there are elements oriented along different spatial directions.

\subsection{Variations in material properties}

Variability in material properties can be specified for density, $\rho$, Young modulus, $E$ and specific fracture energy, $G_{f}$. A given material property $X$ is specified for each element as a function of its mean value, $\bar{X}$, using

$X=\varphi(\gamma) \bar{X}$

where

$\varphi(\gamma)=1-e^{-\left(\frac{X}{\beta}\right)^{\gamma}}$

is a random number with a Weibull distribution and mean value equal to unity. The scale parameter of the distribution is

$\beta=\frac{\bar{X}}{\Gamma\left(1+\frac{1}{\gamma}\right)}$

where $\Gamma$ is the Gamma function (see Ref. [10]). The shape parameter $\gamma$ is computed from a non-linear regression of the experimental data.

The variability is given by the ratio between the standard deviation and the mean value,

$C V(X)=\sqrt{\frac{1}{n} \sum(\varphi(\gamma)-1)^{2}}$

where $n$ is the number of data points.

Following the works by Rios and Riera [29] and by Miguel et al. $[21,22]$, it is assumed here that randomness of the material properties is independent of the model discretization.

\subsection{Implementation in ABAQUS/Explicit}

The LDEM is implemented in ABAQUS/Explicit [7] using 2-node linear-displacement truss elements (T3D2) for the model discretization. Elements are divided into two sets, one for the longitudinal and one for the diagonal elements. The cross-sectional areas and the Young's modulus are computed using equations (1) and (2) and specified via the *SOLID SECTION and *ELASTIC keywords. Similarly, element densities are specified using *DENSITY. Densities are assigned so that, once the model is assembled, equivalent nodal masses are those introduced in Section 2. Densities for the longitudinal and diagonal elements, $\rho_{l}$ and $\rho_{d}$, are computed as functions of element cross-sectional areas and lengths. The resulting formulas are

$\rho_{l}=\frac{\rho}{7.8}$ and $\quad \rho_{d}=\frac{\rho}{5.2}$

Special cases are the nodes on the model surface, which according to the previous procedure, will result with mass deficits due to the absence of neighbour modules. These deficits are compensated by the addition of complementary discrete masses, the values of which are computed depending on the node connectivities. The details about this procedure are in Ref. [17]. Discrete masses are specified via the *MASS keyword.

The non-linear constitutive law is implemented by means of the smeared model due to due [11] via the *BRITTLE CRACKING, *BRITTLE SHEAR and *BRITTLE FAILURE keywords: *BRITTLE CRACKING is set as TYPE $=$ STRAIN and used to set the critical failure strain (point 
A in Fig. 2) and the limit strain (point B in Fig. 2); *BRITTLE SHEAR is only specified to complete the model data, note that, since ModeII fracture is not relevant for bar elements, this datum is not used; and *BRITTLE FAILURE is used to activate the automatic removal of the failed elements from the model once they attain the limit strain.

\section{Experimental tests}

\subsection{Falling-weight impact test}

Falling-weight impact tests were conducted on a $3.2-\mathrm{mm}$ thick sheet of PMMA kindly provided by Ineos Acrylics (Rolle, Switzerland). Tests were carried out according to ASTM D3763 [1] using a Fractovis Gravity Drop CEAST 6789 equipped with a CEAST DAS 4000 data acquisition system. Fig. 3 illustrates a scheme of the test setup. Four experiments were tested using a hemispherical striker of diameter $d=12.7 \mathrm{~mm}$, mass $m=18.49 \mathrm{~kg}$ and nominal velocity $v_{0}=1 \mathrm{~m} / \mathrm{s}$. The diameter of the clamp was $D=76 \mathrm{~mm}$.

Fig. 4(a) and (b) depicts the plots of the striker force vs. time and striker velocity vs. time records, respectively. The curves in Fig. 4(a) show three force peaks. The early peak during the loading stage at $t \approx 0.5 \mathrm{~ms}$ is the so-called "inertial peak", and it is not relevant for the present work (please refer to Kakarala and Roche [14] for a detailed description of the phenomena). The other two force peaks are of interest for this work. They occur at the onset of crack propagation and just before the final failure of the specimen, respectively. The first peak is of magnitude $P_{1} \approx 600 \mathrm{~N}$, it occurs at $t_{1} \approx 1.75 \mathrm{~ms}$ and it exhibits an excellent repeatability for all the specimens. On the other hand, the time and magnitude of the second peak present significant dispersions. Maximum forces are in the $600 \mathrm{~N} \leq P_{2} \leq 830 \mathrm{~N}$ range and they occur along the $6.4 \mathrm{~ms} \leq t_{2} \leq 9.4 \mathrm{~ms}$ range. All force traces present important oscillations just after $t_{1}$, but in every case, they gradually diminish and they almost vanish at $t_{2}$. It is worth to observe the gradual decrement of the slopes of the force vs. time plots as the tests progress. This is because of the gradual reduction of the specimen stiffness with crack propagation.

Fig. 4(b) shows that the striker velocity increases at the beginning of the test, when inertial effects prevail over the specimen stiffness. In every test, the maximum velocity is attained at $t \approx 0.8 \mathrm{~ms}$. From this moment on, striker velocity monotonically decreases until the end of the test. Two inflection points in the velocity records occur at times $t_{1}$ and $t_{2}$, respectively. They are consequences of sudden decrements of the specimen stiffness, which result in instant increments in the striker acceleration (see the changes in the slope

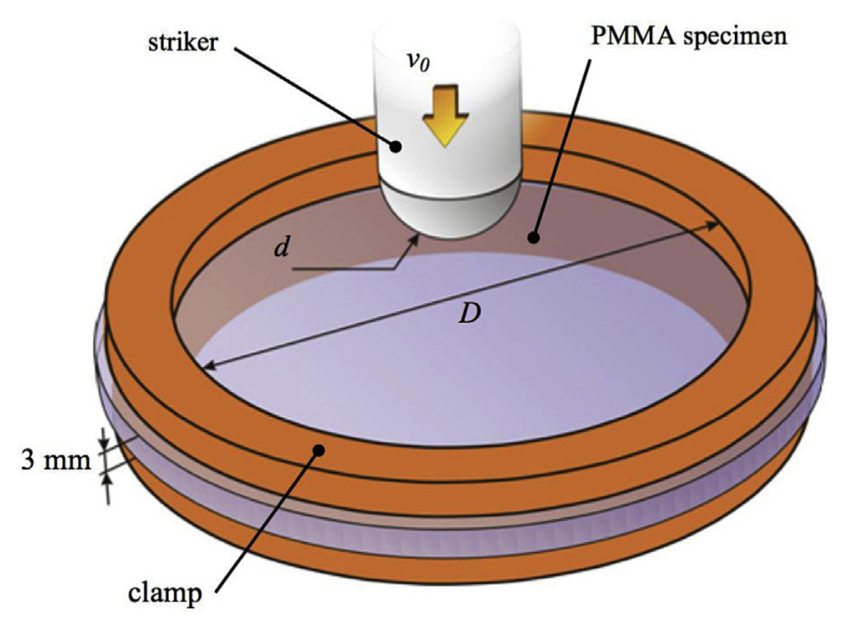

Fig. 3. Schematic of the falling-weight impact test.

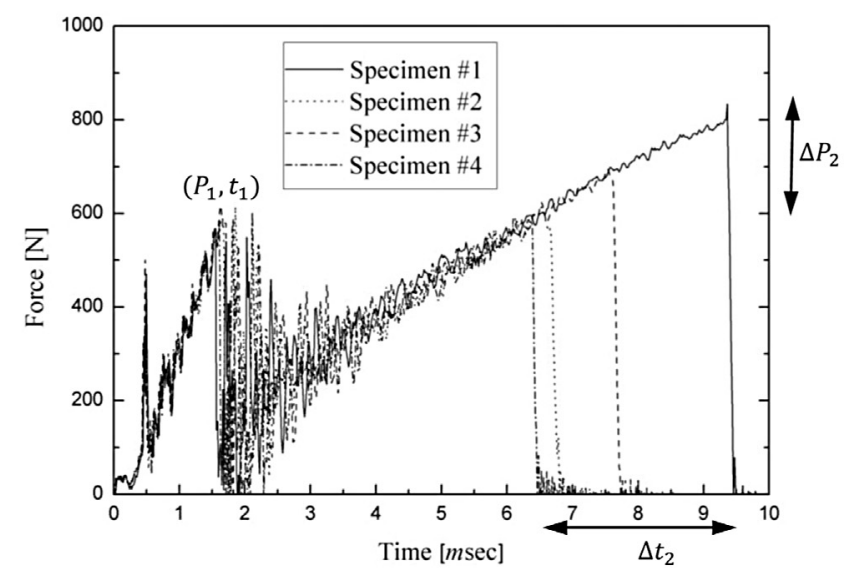

(a)

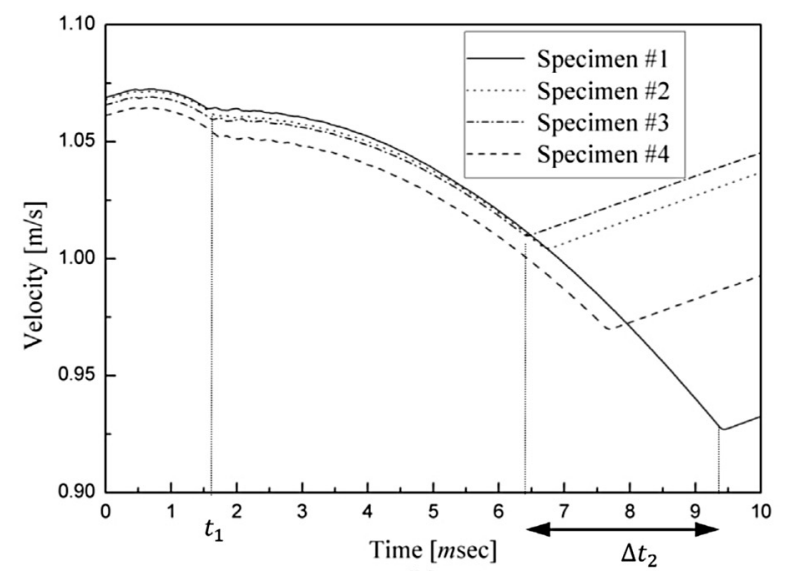

(b)

Fig. 4. Experimental results: (a) force and (b) velocity vs. time records.

of the velocity records). The striker velocity increases after $t_{2}$, once it has gone through the specimen.

Photographs of tested specimens showing the developed crack patterns are depicted in Fig. 5. All the specimens present the same crack pattern: a set of radial cracks joined together by a circular crack. The cracks patterns are characterized in terms of three parameters: the number and length of the radial cracks, and the diameter of the circular crack. The radial crack length is quantified by the diameter of the circumference that best fit the crack vertices. Similarly, a circumference is fitted to the circular crack and used to quantify its diameter. Note that the fitted circumferences are not restricted to be concentric with the perimeter of the circular clamp. The results are compiled in Table 1. It is interesting to see that while there is a significant dispersion in the number of radial cracks has (from 6 to 9), their lengths are nearly constant, with a dispersion less then $6 \%$ with respect the mean value $d_{r}=65.25 \mathrm{~mm}$. The dispersion in the radial crack number may be related to local material inhomogeneities at the impact site. Liu and Liaw [20] reported similar observations for PMMA specimens subjected to low impact energy. The results for the circumferential crack diameter present an important dispersion, in the $38 \mathrm{~mm} \leq d_{c} \leq 54 \mathrm{~mm}$ range.

\subsection{Mechanical characterization of PMMA}

Mechanical properties of PMMA required in simulations were determined by performing uniaxial-tensile experiments and fracture impact tests. 

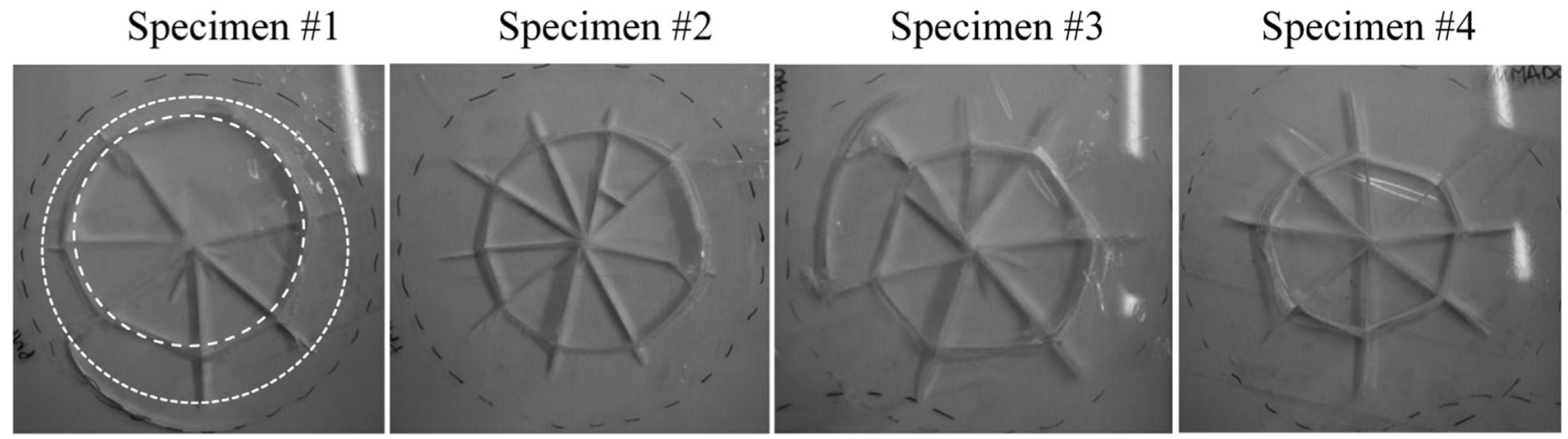

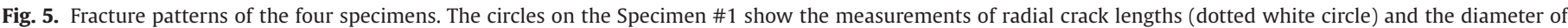
the circular cracks (dashed white circle). The dashed black circles in the photos indicate the perimeters of the specimens.

Tensile tests were conducted according ASTM D638 [2] on Type-V dumb-bell shaped samples at different strain rates. Low rate experiments were carried out at 2,10,50 $\mathrm{mm} / \mathrm{min}$ in an Instron 4467 universal testing machine, while high rate tests were performed at $1800 \mathrm{~mm} / \mathrm{s}$ in a CeastResil 25 pendulum. Three specimens were tested in every case.

Fig. 6 shows typical uniaxial stress-strain curves at different strain rates. It can be observed that the viscous response systematically diminishes with the increment of the strain rate. At the same time, the initial linear response is almost independent of strain rate. As the aim of this work is to simulate the impact response, the behaviour of PMMA was assimilated to that observed at $1800 \mathrm{~mm} /$ s. Therefore, the material was modelled as linear elastic over the complete strain range. The Young modulus value $E=3.35 \mathrm{GPa}$ was computed from the linear part of the curves, $0 \leq \varepsilon \leq 5 \cdot 10^{-3}$. In ac-

Table 1

Geometrical characterization of the experimental crack pattern.

\begin{tabular}{llll}
\hline Specimen & $\begin{array}{l}\text { Number of } \\
\text { radial cracks }\end{array}$ & $\begin{array}{l}\text { Radial crack } \\
\text { length }[\mathrm{mm}]\end{array}$ & $\begin{array}{l}\text { Circular crack } \\
\text { diameter }[\mathrm{mm}]\end{array}$ \\
\hline$\# 1$ & 6 & 69 & 54 \\
$\# 2$ & 8 & 65 & 38 \\
$\# 3$ & 9 & 63 & 48 \\
$\# 4$ & 7 & 64 & 47 \\
\hline
\end{tabular}

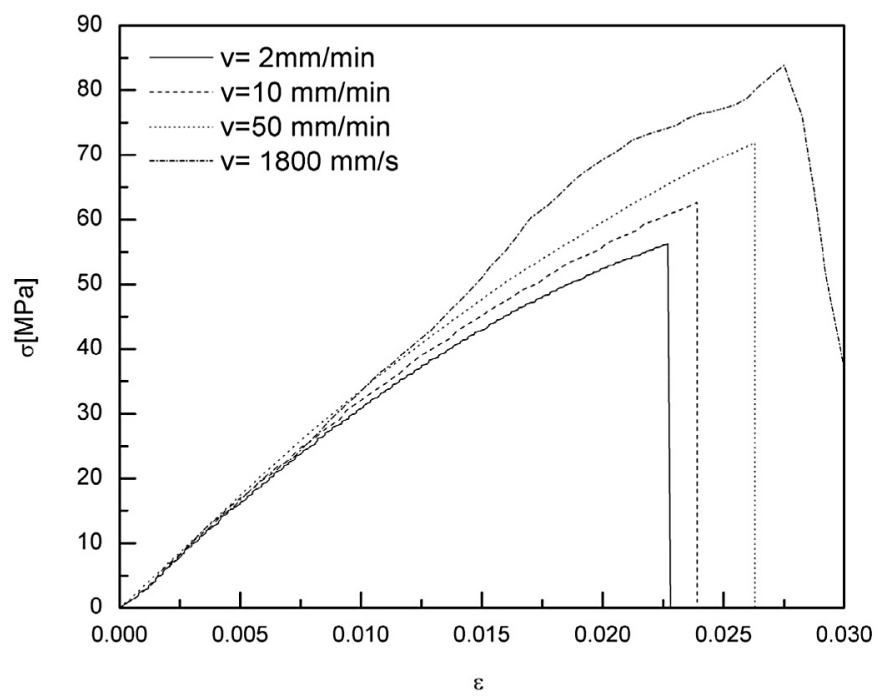

Fig. 6. Tensile tests: material response at different test velocities. cordance with the previous hypothesis, the failure stress was computed as the mean value of the data of the high-velocity tests. This was $\sigma_{f}=75 \mathrm{MPa}$ with a dispersion of $15 \%$.

Fracture impact tests were performed using single notched threepoint-bending specimens according to ISO 17281 [12]. The test machine was the same used in falling-weight impact tests, the striker mass was $3.6 \mathrm{~kg}$ and the span was set $S=24 \mathrm{~mm}$. Fourteen specimens were tested, the dimensions of which were: thickness $B=3 \mathrm{~mm}$, width $6.21 \mathrm{~mm} \leq W \leq 7.26 \mathrm{~mm}$ and initial notch depth $1.9 \mathrm{~mm} \leq a \leq 6.38 \mathrm{~mm}$. Sharp notches were introduced into the samples using a standard notching machine equipped with a razor cut.

Fig. 7 shows the resulting fracture-energy values, $U$, plotted as a function of $B W \phi$ (where $\phi$ is a calibration factor that depends on the specimen geometry, see Ref. [3]). The fracture toughness value $G_{I C}=930 \pm 120 \mathrm{~J} / \mathrm{m}^{2}$ was determined following the standard procedure [12].

The material density was $\rho=1190 \mathrm{~kg} / \mathrm{m}^{3}$.

\section{LDEM model}

Fig. 8 illustrates the LDEM model of the of the impact specimens. It consists in $92.8 \mathrm{~mm} \times 92.8 \mathrm{~mm} \times 3.2 \mathrm{~mm}$ square plate discretized with elements of length $L=0.533 \mathrm{~mm}$. This results in

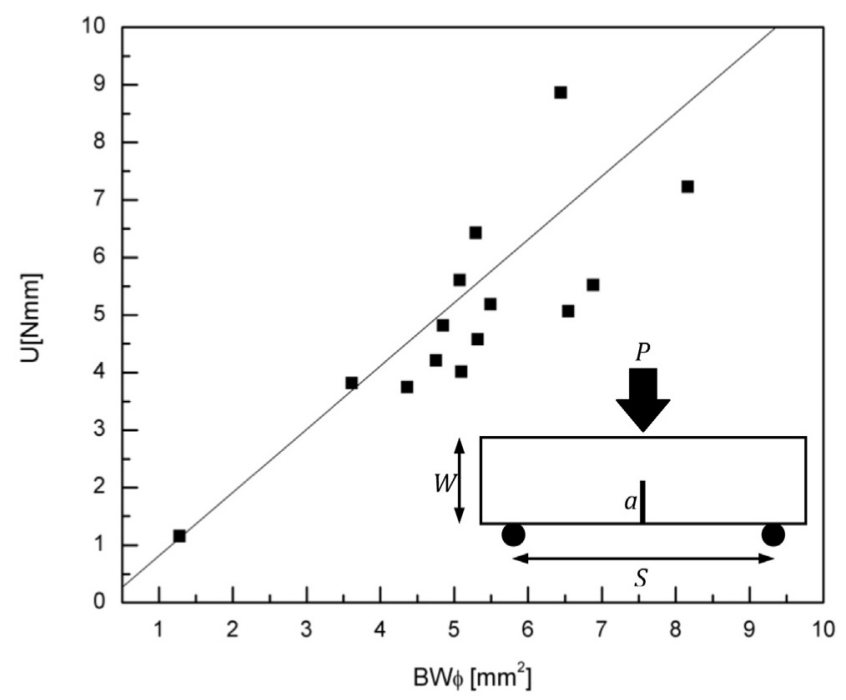

Fig. 7. Results of the impact fracture test. 


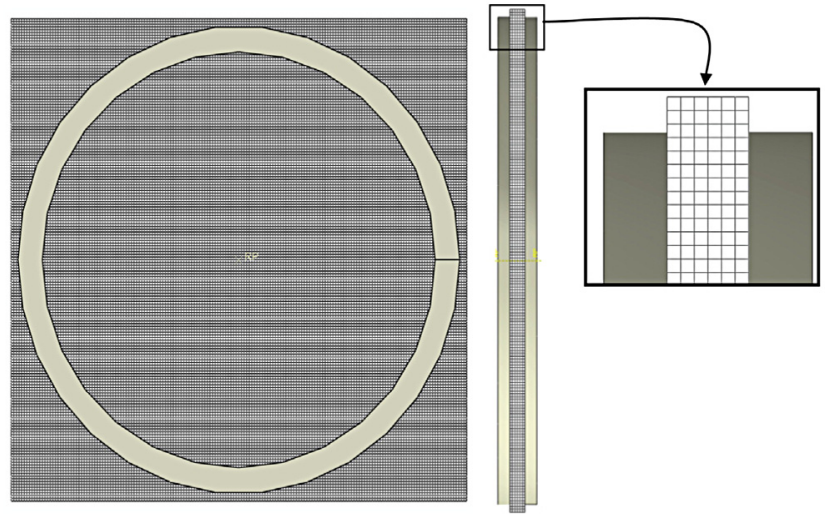

Fig. 8. LDEM model of the impact test.

six modules across the specimen thickness. Model consists of approximately $2.510^{6}$ elements with $1.210^{6}$ degrees of freedom.

The clamp is modelled using two rigid rings, one on each side of the plate (see Fig. 3). Both rings are anchored to prescribe null displacements and rotations. The inner and outer diameters of the rings are $76 \mathrm{~mm}$ and $90 \mathrm{~mm}$ respectively. Rings are modelled as rigid bodies via the *RIGID BODY. No-slip contact boundary conditions are specified between the rings and the plate via *SURFACE INTERATION, *FRICTION, ROUGH and *SURFACE BEHAVIOR, PRESSURE-OVERCLOSURE=HARD keywords. The hemispherical striker is modelled as a *RIGID BODY with mass $m=18.49 \mathrm{~kg}$. The striker displacement is only allowed in the direction perpendicular to the plate surface. The contact boundary condition between the striker and the plate is specified as *FRICTION, 0; this is, frictionless. According to the results in Fig. 4(b), the initial striker is specified $v_{0}=1.065 \mathrm{~m} / \mathrm{s}$. The velocity and force as functions of time are reported at the striker reference point.

The impact test is modelled for two sets of material properties:

\section{a) Homogeneous material}

Parameters of the non-linear constitutive law are computed from mean values of the experimental data in Section 3.2 and the adopted element length. The element stiffnesses are $E_{l}^{A}=375.47 \mathrm{~N} / \mathrm{m}$ and $E_{d}{ }^{A}=195.10 \mathrm{~N} / \mathrm{m}$, see equations (1) and (2); the specific fracture energy $G_{f}=1034 \mathrm{~N} / \mathrm{m}$; the equivalent fracture areas $A_{d}{ }^{f}=0.03878 \mathrm{~mm}^{2}$ and $A_{l}{ }^{f}=0.05171 \mathrm{~mm}^{2}$, see equations (7) and (8); the critical failure strain, $\varepsilon_{p}=0.01818$ with $R_{f}=31.45$, see equation (9); and the limit strain $\varepsilon_{r}=0.02238$ with $K_{r}=1.23$, see equation (11).
In addition to the analysis of the impact problem, the model with the homogeneous materials was used to solve the eigenvalue problem to compute the natural frequency for the specimen freeoscillations. These results are used to assess the quality of the LDEM model. The eigenvalue problem was solved using Abaqus/Standard procedures by means of the *STEP, PERTURBATION and *FREQUENCY commands.

\section{b) Non-homogeneous material}

LDEM parameters are set the same of the homogeneous case, with the exception of the fracture toughness, which is specified using a Weibull distribution of probability, as it is detailed in Section 2.3. The mean value for the distribution is $G_{f}=1034 \mathrm{~N} / \mathrm{m}$, and three values for the variability are considered, $C V\left(G_{f}\right)=0.10,0.25$ and 0.50 . The strategy for the setting of the $G_{f}$ is illustrated in Fig. 9: the different values of $G_{f}$ are accompanied by consistent adjustments of the critical and the limit strains, $\varepsilon_{p}$ and $\varepsilon_{r}$, such that the values of $R_{f}$ and $K_{r}$ are keep constant, see equations (10) and (12).

The time increment was set $\Delta t=9 \cdot 10^{-8} \mathrm{~s}$, which satisfies both, the LDEM stability condition in equation (13) and the critical time increment required by Abaqus.

\section{Results}

\subsection{Free oscillations}

Results of the eigenvalue analysis are given in Table 2 and Fig. 10. Frequency results are compared to those computed using the Rayleigh-Ritz method for a circular clamped disk [36]. It can be observed that there is a good agreement between the two sets of results. This evidences the effectiveness of the model to properly represent the model stiffness and the spatial mass distribution. No attempt was made to compensate the differences via the calibration of the material properties.

\subsection{Falling-weight impact tests}

Results of the simulations of the impact test of the homogeneous specimen are shown in Figs. 11 and 12. Fig. 11 presents the plots of the time evolutions of the striker force and velocity. Also included in these plots are the envelopes of the experimental results. Fig. 11(a) allows observing that the LDEM captures the first force peak at $t_{1}$ very accurately, within the dispersion of the experimental results. Like in experiments, LDEM results present important oscillations after the first force peak, but in contrast to experiments, the oscillations in the numerical results do not diminish

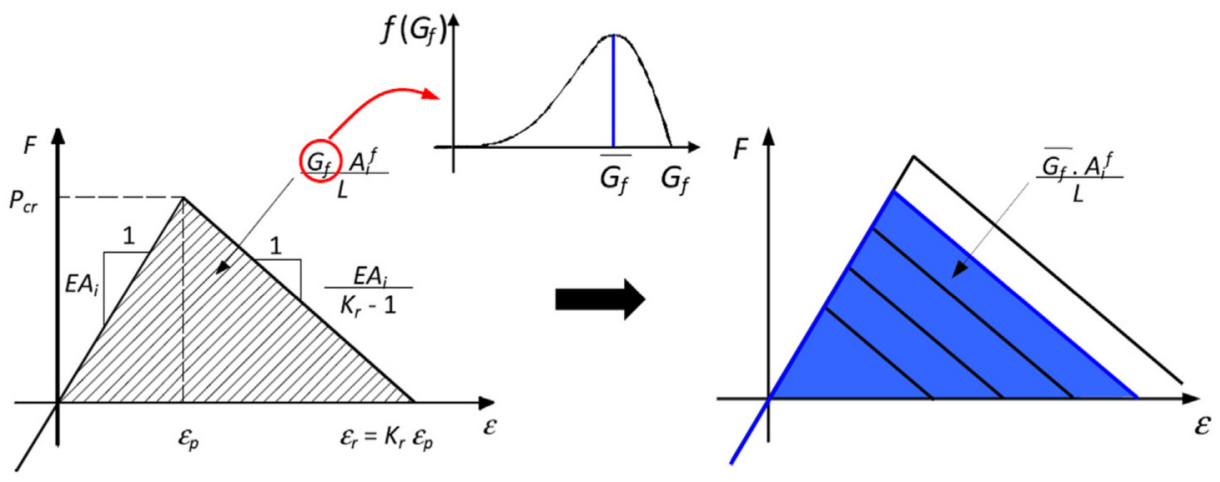

Fig. 9. Strategy for the setting of the Weibull distribution for the fracture toughness. 
Table 2

Modes of the free oscillations of the homogeneous disks.

\begin{tabular}{lccc}
\hline \multicolumn{4}{l}{ Free-oscillation frequencies $[\mathrm{kHz}]$} \\
\hline Mode & LDEM & Rayleigh-Ritz & Difference \\
\hline 1 & 1.46 & 1.61 & $-9.6 \%$ \\
2 & 3.04 & 3.35 & $-9.3 \%$ \\
3 & 4.85 & 5.51 & $-12.0 \%$ \\
4 & 5.51 & 6.29 & $-12.3 \%$ \\
5 & 11.85 & 14.05 & $-15.6 \%$ \\
\hline
\end{tabular}

with time. This difference might be attributed to the some damping capacity of the striker and/or the specimen, which have not been considered in the LDEM model. It is important to note that, although the oscillations, the mean value of the force vs. time plot along during $t_{1} \leq t \leq t_{2}$ presents the same slope as it experimental counterpart. Moreover, the predicted time to failure, $t_{2} \approx 7.5 \mathrm{~ms}$, lies well within the experimental envelope. These are indicators that the model is able to capture the sequence of events conducting to the specimen failure. In an attempt to attenuate the effect of the oscillations, LDEM results for $t>t_{1}$ are filtered using a lowpass filter for the frequencies of the free-oscillations computed in Section 5.1. Results for the filters with $f_{1}$ and $f_{2}$ are given in Fig. 11(a). It can be observed that the filter for $f_{1}$ has a noticeable effect. Filtered LDEM results exhibit amplitudes of the same order of their experimental counterpart. Filtering with frequencies higher to $f_{1}$ has no noticeable effects. These results indicate that the specimens oscillate mainly in the first (lowest) mode, what it is coherent with the shape of the displacement field that is induced to the specimen in the beginning of the test.

LDEM results for the time evolution of the striker velocity is in very good agreement with the experiments; see Fig. 11(b). The LDEM output is within the narrow envelope of the experimental results up to the time of the first force peak. The model fails to catch the inflection in the velocity curve at $t_{1}$, and from this moment on, the velocity is underestimated by approximately $2 \%$. The predicted striker velocity after failure lies well within the experimental envelope. In contrast to force results, there are no oscillations in velocity results.

Fig. 12 presents the time evolution of the energies and the specimen fracture pattern. The time evolution of the energies in Fig. 12(a) is completely coherent with the above results. The strain energy monotonically increases up to $t_{2}$, the moment in which the specimen loses its load carrying capacity due to final failure. On the other hand, the specimen kinetic and dissipated damage energies are negligible before $t_{1}$. Increments in these magnitudes after $t_{1}$ are associated to the onset and subsequent crack propagation. Both, damage and kinetic energies exhibit sudden increments at $t_{2}$. Increment in dissipated damage energy is due to rapid crack propagation, while increment in the kinetic energy is due to

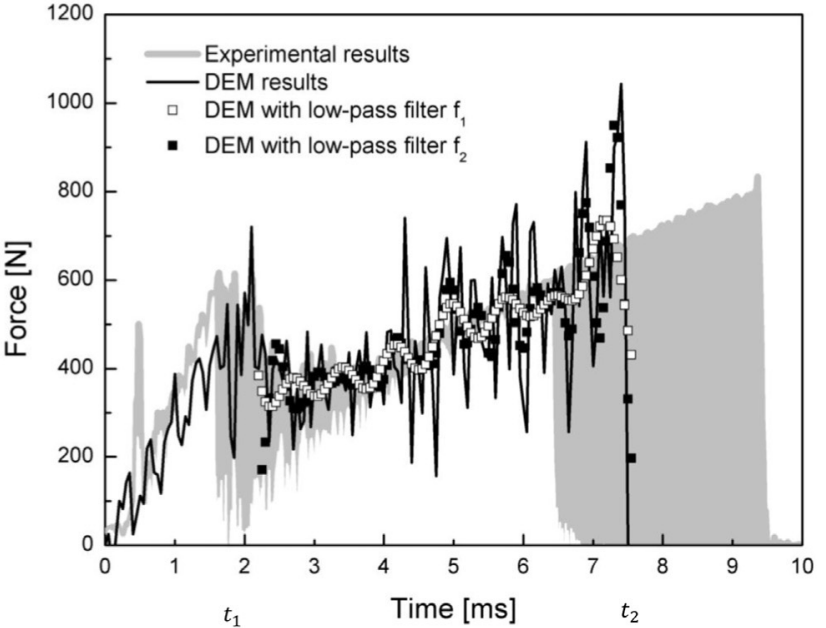

(a)

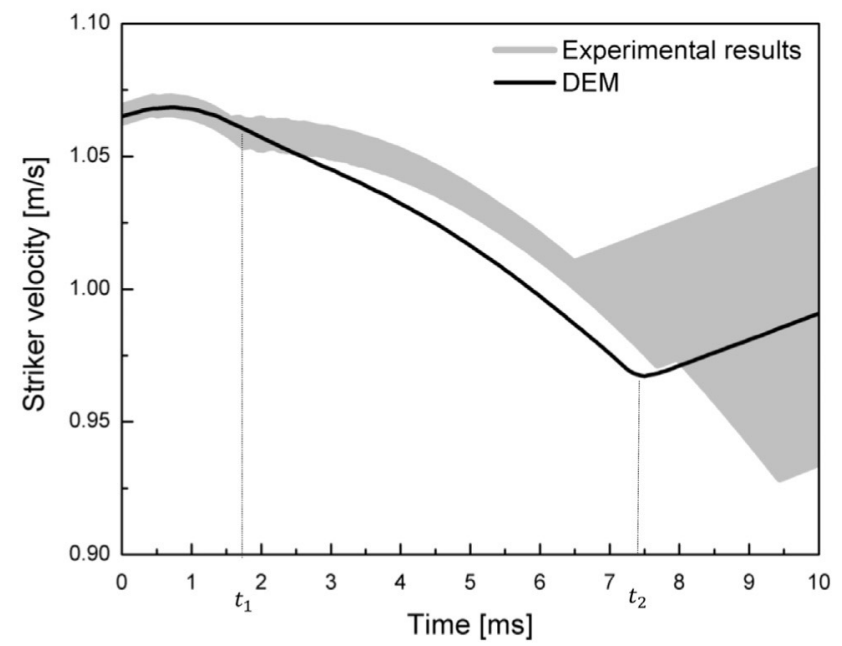

(b)

Fig. 11. LDEM results for the homogeneous specimen: (a) force and (b) velocity vs. time records.

specimen pieces moving apart after failure. The predicted fracture pattern in Fig. 12(b) is in good agreement with the photographs of the tested specimens in Fig. 5. The model nucleates five radial cracks, which are just below the low bound of the experimental results. The radial crack pattern is uniform, like in the experiments, but the crack length, $d_{r} \approx 57 \mathrm{~mm}$ is smaller than that experimentally measured. The diameter of the circumferential crack $d_{c} \approx 42 \mathrm{~mm}$ is within the experimental range.
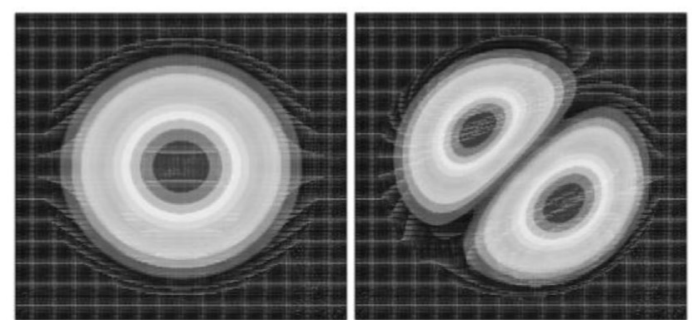

$f_{1}=1.46 \mathrm{KHz}$

$f_{2}=3.04 \mathrm{KHz}$
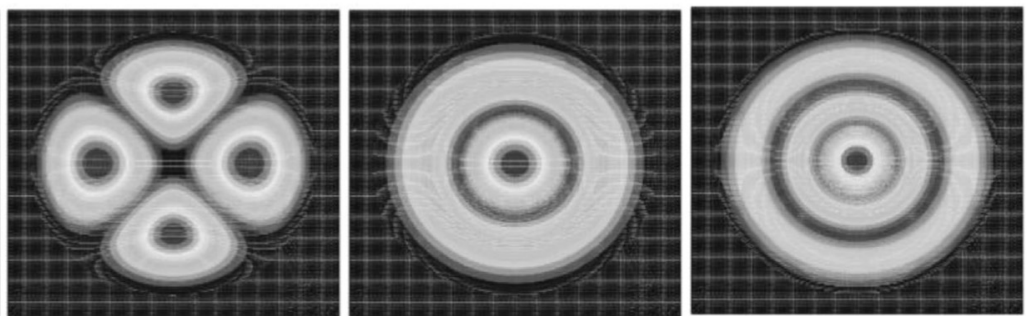

$f_{3}=4.85 \mathrm{KHz}$

$f_{5}=11.85 \mathrm{KHz}$

Fig. 10. Modes of the free oscillations of the homogeneous specimen. 


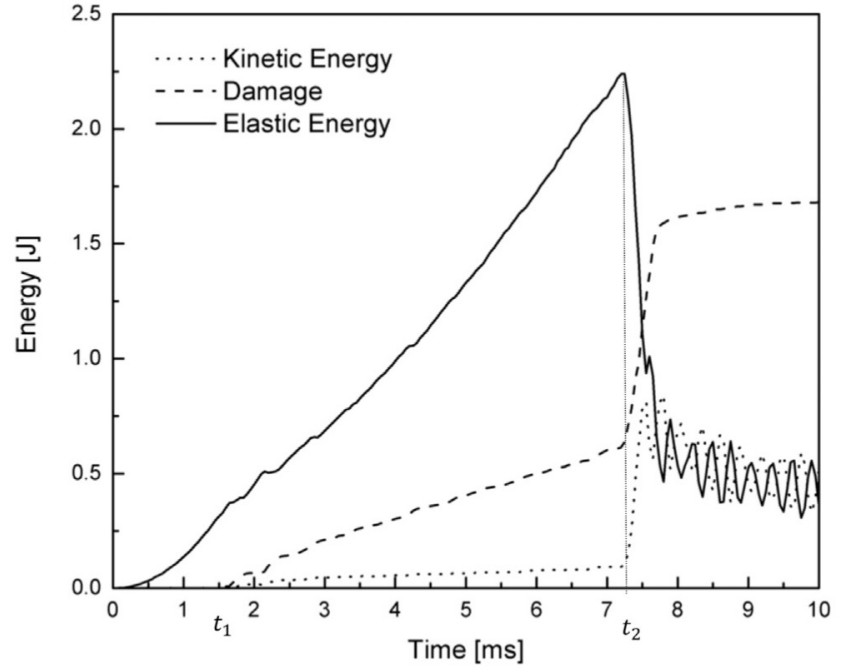

(a)

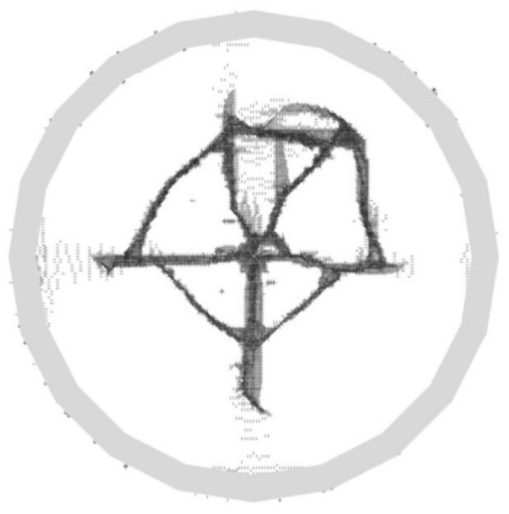

(b)

Fig. 12. LDEM results for the homogeneous specimen: (a) time evolution of the strain, kinematic and damage energies and (b) fracture pattern. The dotted circle is for the measurement of the circumferential crack radio.

The results for the heterogeneous specimens are given in Figs. 13-15. Fig. 13 illustrates the time evolution of the striker force and the velocity for the three variabilities in $G_{f}$ distribution. Four tests with different random $G_{f}$ fields were simulated for each case. There are also given in the figure the envelopes for the experimental results. Force results are filtered for the free-oscillation frequencies using the same procedure as for the homogeneous case. It can be observed in Fig. 13(a) that the simulations with the three levels of variability capture the first peak: the simulations with $C V\left(G_{f}\right)=10 \%$ and $25 \%$ provide accurate predictions for $P_{1}$ and $t_{1}$ while the simulations with $C V\left(G_{f}\right)=50 \%$ properly predicts $t_{1}$, but underestimates $P_{1}$. The simulations with $C V\left(G_{f}\right)=10 \%$ and $25 \%$ present similar behaviours, close to that of the homogeneous case for $t_{1} \leq t \leq t_{2}$, whereas $C V\left(G_{f}\right)=50 \%$ can be assimilated to the lower bound of the numerical results. This last observation is coherent with the results for the velocity time evolution in Fig. 13(b). Velocity vs. time plots for all the simulations with $C V\left(G_{f}\right)=10 \%$ and $25 \%$ present practically no dispersion and they are coincident to that of the homogeneous case; see Fig. 11(b). In the case $C V\left(G_{f}\right)=50 \%$, three out of the four simulations result with velocities higher than those of their counterparts with lower variabilities in $G_{f}$. In every case, the numerical results for velocity are in excellent agreement with experimental ones.

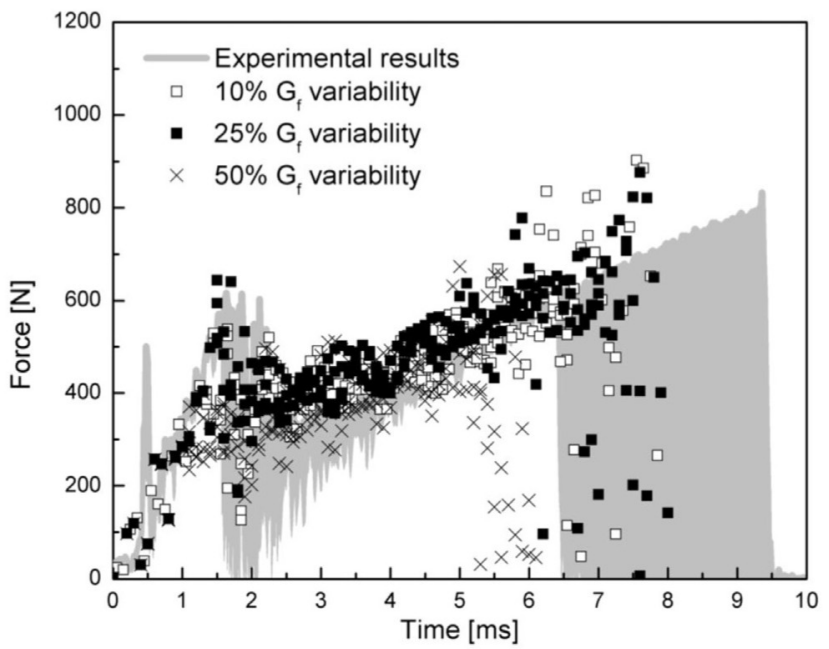

(a)

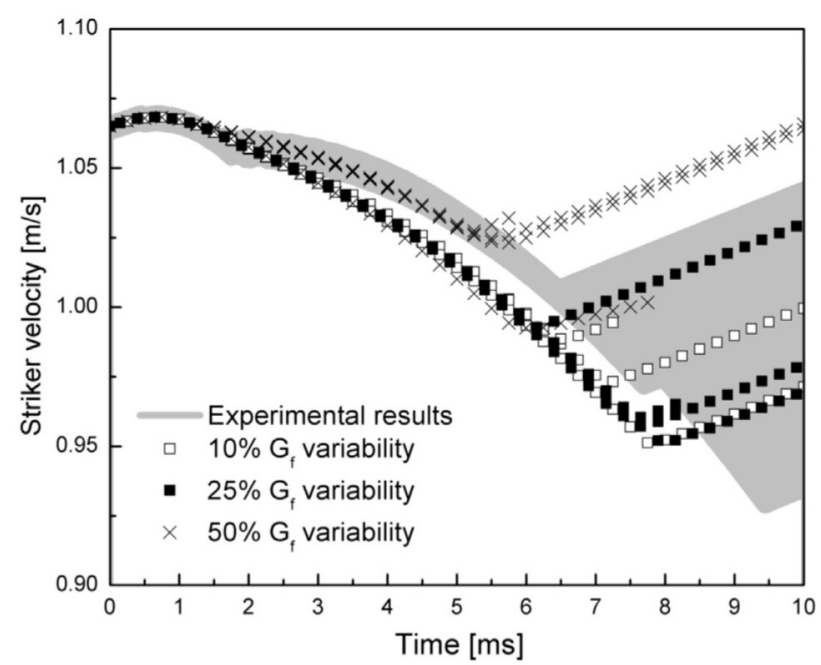

(b)

Fig. 13. LDEM results for the heterogeneous specimen: (a) force and (b) velocity vs. time records for three values of variability of the material fracture energy.

It can be observed from Fig. $13(\mathrm{a})$ that $C V\left(G_{f}\right)=10 \%$ and $25 \%$ predicts failure times $\left(t_{2}\right)$ between $6 \mathrm{~ms}$ and $8 \mathrm{~ms}$. This range is almost completely comprised within the experimental envelope. In contrast, the case $C V\left(G_{f}\right)=50 \%$ consistently predicts that the final failure occurs earlier than it is observed in the experiments. It is worth noting that, due to the absence of oscillations, the velocity vs. time plot is the best means to measure the time to failure.

Results for the energy time evolution are shown in Fig. 14. It can be observed that in every case, the results are very smooth and they present no oscillations for $t \leq t_{2}$. So, these plots are also accurate means to retrieve the time to failure. Consistently with the results above, the sets of results for $C V\left(G_{f}\right)=10 \%$ and $25 \%$ are nearly coincident, while the results for $C V\left(G_{f}\right)=50 \%$ slightly differ. The damage energy plot is of particular interest, as its departure from zero allows retrieving $t_{1}$, the time for fracture initiation.

Fig. 15 depicts snapshots of the fracture patterns at the radial cracks achieve their maximum length, i.e., just before the nucleation of the circular crack. This is found to happen at $t_{2}$. Nucleation and propagation of the circular cracks occur almost instantaneously, not later than $0.4 \mathrm{~ms}$ to $0.5 \mathrm{~ms}$ after $t_{2}$. The snapshots with the final fracture patterns are depicted in Fig. 16. 


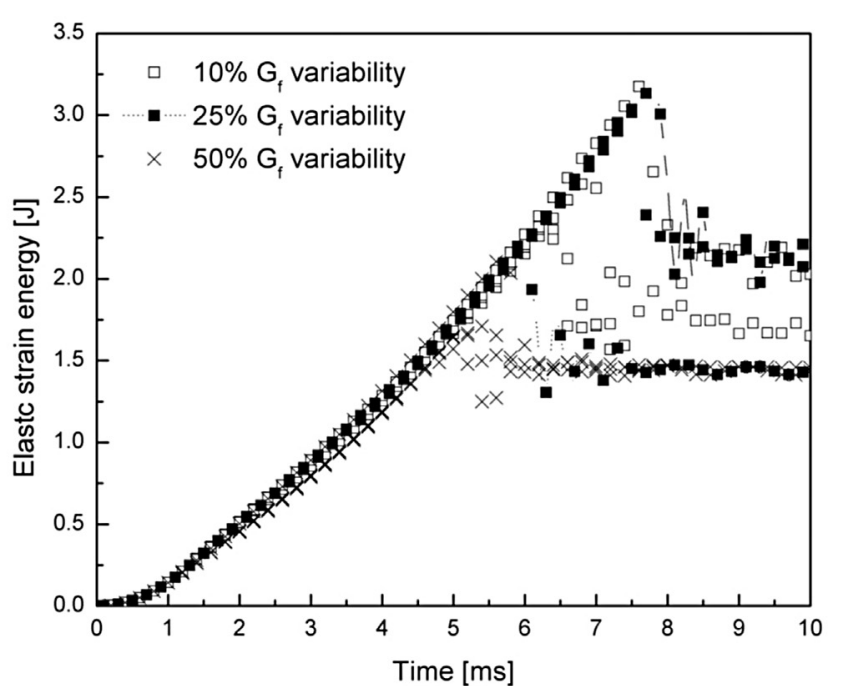

(a)

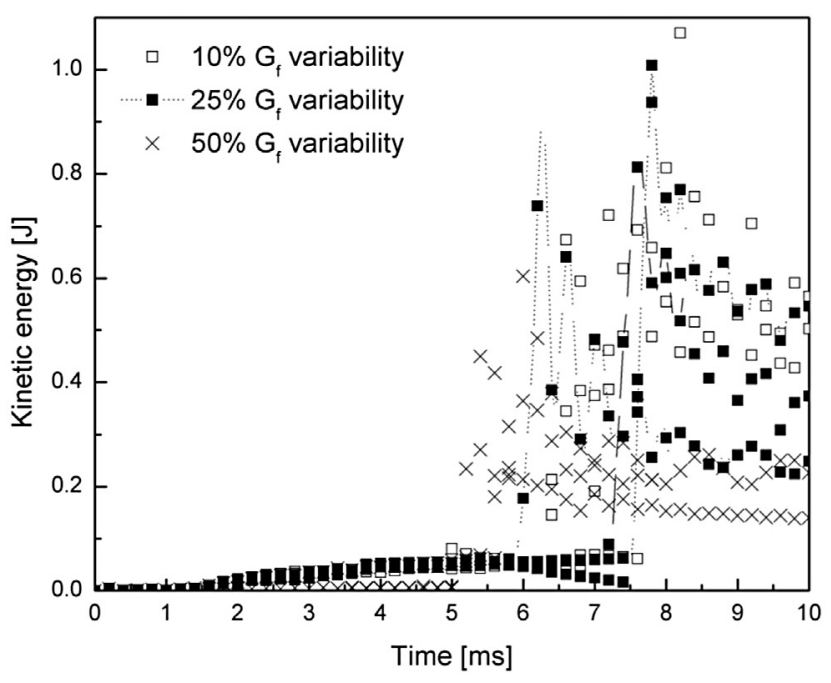

(b)

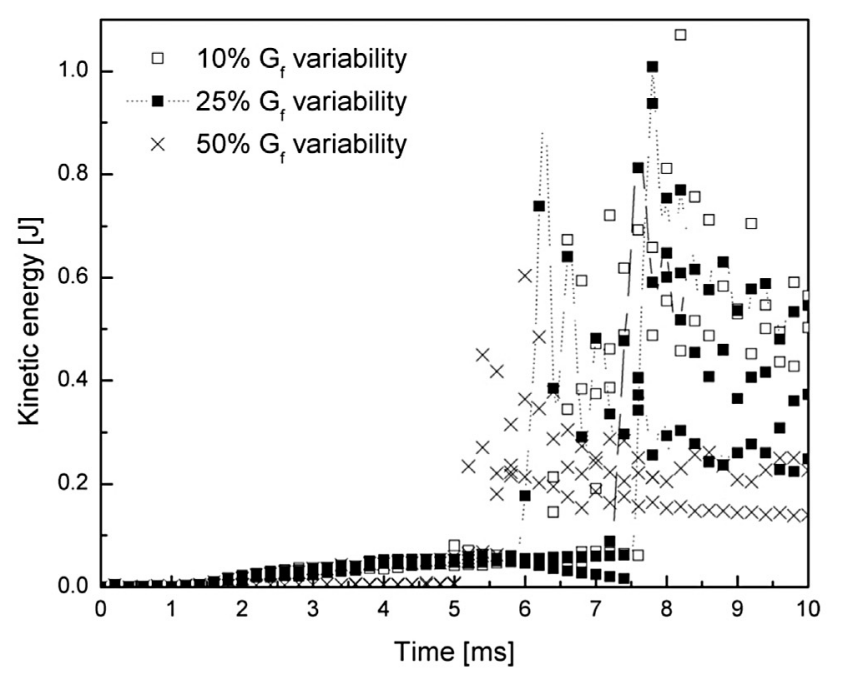

(c)

Fig. 14. LDEM results for the heterogeneous specimen: time evolution of the (a) strain energy, (b) dissipated damage energy and (c) kinetic energy for three values of variability of the material fracture energy.
Table 3 compiles the data of the geometrical characterization of the LDEM crack patterns. It can be observed that the heterogeneity in the fracture energy does not have a major effect on the number of radial cracks, which ranges from 4 to 6 in all the cases. Maximum number of cracks in the simulations attains the minimum number of cracks observed in experiments. Similarly, the variability in $G_{f}$ has not a strong influence on the radial crack lengths. As in the homogeneous case, the simulations consistently underestimate radial crack lengths. On the other hand, circular crack diameters, replicating the experimental observations. Most of the crack-diameter results for $C V\left(G_{f}\right)=10 \%$ and $25 \%$ lie within the bottom half of the experimental measurements (see Table 1), while the simulations for $C V\left(G_{f}\right)=50 \%$ conduct to predictions that are smaller than their experimental counterparts.

\section{Conclusions}

This paper presents a lattice discrete element method (LDEM) to model the falling-weight impact response of polymethylmethacrylate (PMMA) specimens. The LDEM is based on a formulation that accounts for material damage that in turn allows cracks to nucleate and to propagate spontaneously, without the use of initial cracks or special elements. The LDEM has been implemented using Abaqus/Explicit. This not only makes the implementation very versatile in terms of the model pre and post processing, but it allows exploiting the Abaqus capabilities for contact mechanics, eigenvalue analysis and for the inclusion of rigid parts into the model assembly.

Computational analyses are complemented with experimental tests. The mechanical characterization of the PMMA is performed using uniaxial tensile and fracture impact tests. Experimental results of a set of falling-weight biaxial impact tests on circular specimens are used to validate the numerical models. LDEM simulations are, in every case, of predictive nature. This is, material properties are neither left open for calibration nor used to adjust the numerical results. Simulations are carried for specimens with homogeneous and heterogeneous material fracture energy.

LDEM has the capability to capture all the main events during the experiment: the initial elastic specimen loading prior to the crack initiation, the nucleation and propagation of the radial cracks as the test progresses, and the final failure after the rapid propagation of a circular crack that joins the radial cracks together. Numerical and experimental fracture patterns are in good agreement. Consistent with experimental results, simulations show that the variability in the fracture energy has no significant effects on the number and length of the radial cracks. Moreover, the dispersions of the diameters of the circular cracks are important in both simulations and experiments.

Forces responses from the simulations exhibit larger oscillations than their experimental counterparts. This difference is attributed to the damping capacities of the striker and/or the material, which are not included in the numerical model. A low-pass filter for the specimen free-oscillation-frequencies is introduced to successfully remove these oscillations.

The LDEM predictions of the time histories of the striker force and velocity and of the strain, damage and kinetic energies of the disk are in very good agreement with those retrieved from the experimental results. Evolutions of these variables during the tests show very good correlations with the sequence of events that occur during the tests. Energy results allow having a better insight about the failure mode of the specimens. A variability of up to $25 \%$ in the PMMA fracture energy results in predictions for the specimen failure that lie within the experimental envelope.

The lattice LDEM introduced in this work has shown excellent capabilities to deal with complex impact fracture problems. The 

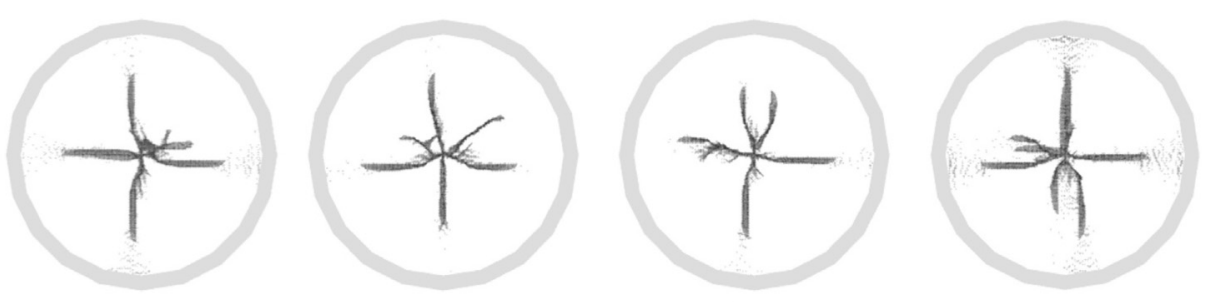

$10 \%$ variability in $G_{f}$
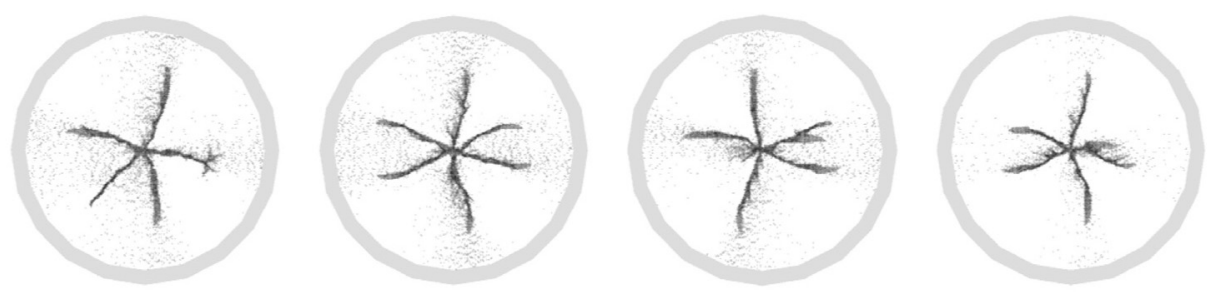

$25 \%$ variability in $G_{f}$
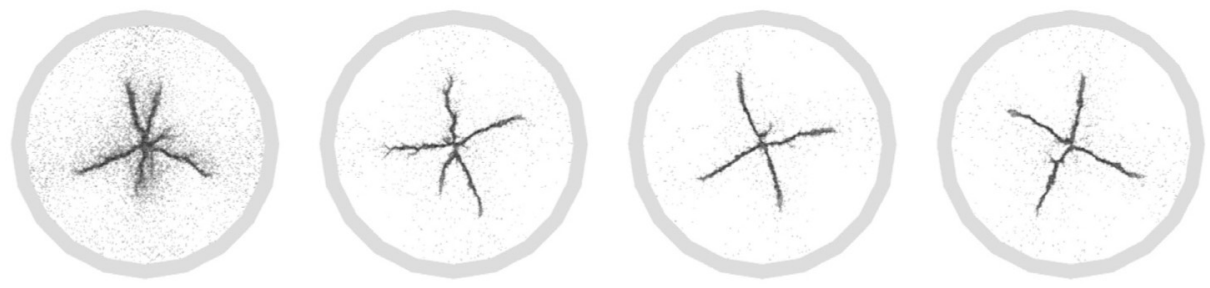

$50 \%$ variability in $G_{f}$

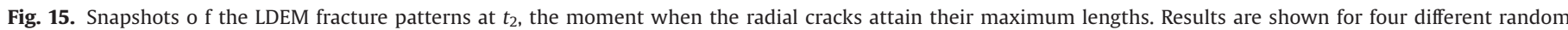
specimens for each of the variations in the fracture energy.
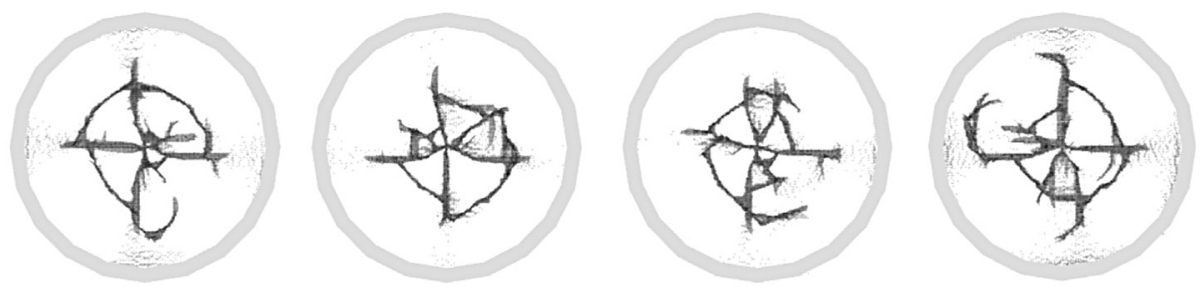

$10 \%$ variability in $G_{f}$
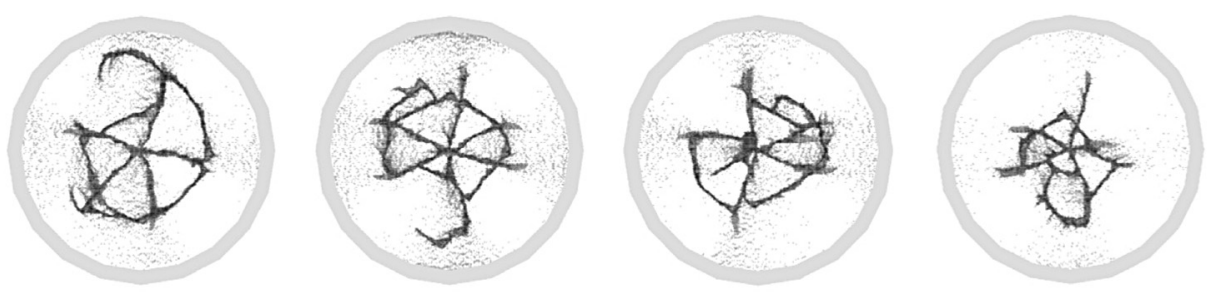

$25 \%$ variability in $G_{f}$
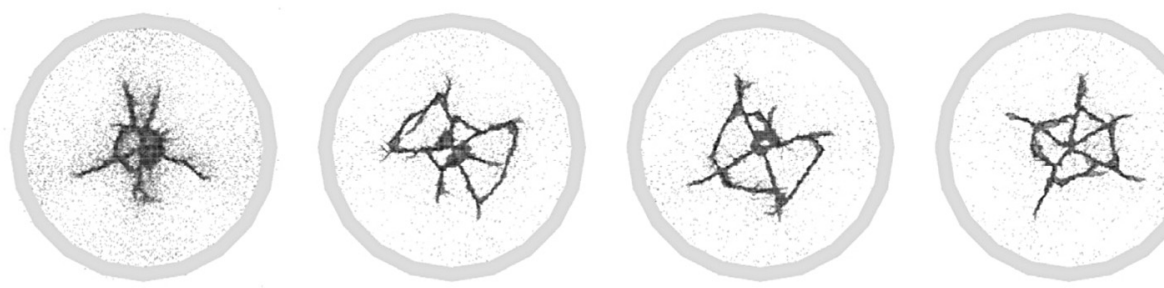

$50 \%$ variability in $G_{f}$

Fig. 16. Snapshots of the LDEM final fracture patters. Results are shown for four different random specimens for each of the variations in the fracture energy. 
Table 3

Geometrical characterization of the LDEM crack patterns.

\begin{tabular}{lllllllllllllll}
\hline & \multicolumn{3}{l}{$\begin{array}{l}\text { Number of } \\
\text { radial cracks }\end{array}$} & \multicolumn{3}{l}{$\begin{array}{l}\text { Radial crack } \\
\text { length [mm] }\end{array}$} & \multicolumn{5}{l}{$\begin{array}{l}\text { Circular crack } \\
\text { diameter [mm] }\end{array}$} \\
\hline Homogeneous & 5 & & & 58.5 & & & & 42 & & & \\
$\mathrm{CV}\left(G_{f}\right)=10 \%$ & 4 & 6 & 5 & 6 & 55.7 & 52.7 & 52.3 & 56.2 & 44 & 38 & 40 & 38 \\
$\mathrm{CV}\left(G_{f}\right)=25 \%$ & 5 & 6 & 6 & 5 & 53.6 & 54.7 & 53.4 & 46.8 & 46 & 42 & 42 & 32 \\
$\mathrm{CV}\left(G_{f}\right)=50 \%$ & 5 & 6 & 4 & 5 & 46.3 & 48.8 & 47.5 & 49.7 & 16 & 34 & 32 & 26 \\
\hline
\end{tabular}

method is versatile, and its implementation into Abaqus allows easily extending it to deal with other material behaviours and more complex problems. Natural extensions to this work are to investigate the performance of constitutive laws with different softening curves, like those proposed by Elices et al. [9] and Murphy and Ivankovic [23] for PMMA in the context of the cohesive zone models, and to account for damping effects. It might result also of interest to couple LDEM with finite element method, what would allow combining the capabilities of both methods in a single model.

\section{Acknowledgements}

The authors wish to express their gratitude for the financial support of ANPCyT, CONICET, and the Universidad de Mar del Plata of Argentina, and CNPq and CAPES of Brazil.

\section{References}

[1] ASTM Standard D3763. Standard test method for high speed puncture properties of plastics using load and displacement sensors. West Conshohocken, PA, USA: ASTM International; 2002.

[2] ASTM Standard D638-02a. Standard test method for tensile properties of plastics. West Conshohocken, PA, USA: ASTM International; 2002.

[3] Bakker A. Compatible compliance and stress intensity expressions for the standard three-point bend specimen. Int J Fatigue Fract Eng Mater Struct 1990;13(2):145-54.

[4] Belytschko T, Chen H, Xu J, Zi G. Dynamic crack propagation based on loss of hyperbolicity with a new discontinuous enrichment. Int J Numer Methods Eng 2003;58:1873-905.

[5] Cundall P, Hart R. Numerical modelling of discontinua. Eng Comput 1992;9(2):101-13.

[6] Dalguer LA, Irikura K, Riera JD, Chiu HC. The importance of the dynamic source effects on strong ground motion during the 1999 Chi-Chi, Taiwan, earthquake: brief interpretation of the damage distribution on buildings. Bull Seismol Soc Am 2001;91(5):1112-27.

[7] Dassault Systèmes, Simulia. Abaqus 6.13 user manual. 2013, Providence, RI, USA.

[8] Fraternali F, Angelillo M, Fortunato A. A lumped stress method for plane elastic problems and the discrete-continuum approximation. Int J Solids Struct 2002;39:6211-40.

[9] Elices M, Guinea GV, Gómez J, Planas J. The cohesive zone model: advantages, limitations and challenges. Eng Fract Mech 2002;69(2):137-63. doi:10.1016 S0013-7944(01)00083-2.

[10] Hahn GJ, Shapiro SS. Statistical models in engineering. New York: John Wiley \& Sons; 1994.

[11] Hillerborg A, Modeer M, Peterson PE. Analysis of crack formation and crack growth in concrete by means of fracture mechanics and finite elements. Cem Concr Res 1976;6:773-82.
[12] ISO 17281. Plastics - determination of fracture toughness $\left(\mathrm{G}_{\mathrm{IC}}\right.$ and $\left.\mathrm{K}_{\mathrm{IC}}\right)$ at moderately high loading rates $(1 \mathrm{~m} / \mathrm{s})$. Geneva, Switzerland: International Organization for Standardization; 2002.

[13] Iturrioz I, Miguel LFF, Riera JD. Dynamic fracture analysis of concrete or rock plates by means of the discrete element method. Lat Am J Solids Struct 2009;6:229-45

[14] Kakarala S, Roche J. Experimental comparison of several impact test methods. In: Kessler SL editor.. Instrumented impact testing of plastics and composite materials STP936. Houston: ASTM International; 1986. p. 144-62.

[15] Kessler SL. Instrumented impact testing of plastics and composite materials STP936. Houston: ASTM International; 1986.

[16] Kosteski L, Iturrioz I, Batista RG, Cisilino AP. The truss-like discrete element method in fracture and damage mechanics. Eng Comput 2011;28(6):765-87. doi:10.1108/02644401111154664.

[17] Kosteski L. Application of the truss-like discrete element method to the study of collapse. [PhD thesis], Porto Alegre, Brazil: Universidade Federal do Rio Grande do Sul; 2012 <http://www.bibliotecadigital.ufrgs.br/da.php?nrb=000860803 \&loc $=2012 \& \mathrm{l}=6 \mathrm{a} 15 \mathrm{cf} 4 \mathrm{e} 12 \mathrm{bb} 528 \mathrm{e}>$, [in Portuguese].

[18] Kosteski LE, Riera JD, Iturrioz I, Singh RK, Kant T. Analysis of reinforced concrete plates subjected to impact employing the truss-like discrete element method. Fatigue Fract Eng Mater Struct 2014;doi:10.1111/ffe.12227.

[19] Kupfer HB, Gerstle KH. Behaviour of concrete under biaxial stresses. Je Eng Mech Div Am Soc Civ Eng 1973;99(4):853-66.

[20] Liu Y, Liaw B. Drop-weight impact tests and finite element modeling of cast acrylic plates. Polym Test 2009;28(6):599-611. doi:10.1016/j .polymertesting.2009.04.008.

[21] Miguel FL, Riera JD, Iturrioz I. Influence of size on the constitutive equations of concrete or rock dowels. Int J Numer Anal Methods Geomech 2008;32(15):1857-81.

[22] Miguel FL, Iturrioz I, Riera JD. Size effects and mesh independence in dynamic fracture analysis of brittle materials. Comput Methods Eng Sci 2010;56(1):1-16.

[23] Murphy N, Ivankovic A. The prediction of dynamic fracture evolution in PMMA using a cohesive zone model. Eng Fract Mech 2005;72(6):861-75. doi:10.1016/ j.engfracmech.2004.08.001.

[24] Nayfeh AH, Hefzy MS. Continuum modeling of three-dimensional truss-like space structures. AIAA J 1978;16(8):779-87.

[25] Pandolfi A, Ortiz M. An efficient adaptive procedure for three-dimensional fragmentation simulations. Eng Comput 2002;18(2):148-59. doi:10.1007/ s003660200013.

[26] Rinaldi A, Lai YC. Statistical damage theory of 2D lattices: energetics and physical foundations of damage parameter. Int J Plast 2007;23:1769-825.

[27] Rinaldi A, Krajcinovic D, Peralta P, Lai YC. Lattice models of polycrystalline microstructures: a quantitative approach. Mech Mater 2008;40:17-36.

[28] Riera JD, Iturrioz I. Discrete element model for evaluating impact and impulsive response of reinforced concrete plates and shells subjected to impulsive loading. Nucl Eng Des 1998;179:135-44.

[29] Rios RD, Riera JD. Size effects in the analysis of reinforced concrete structures. Eng Struct 2004;26:1115-25

[30] Rocha MM, Riera JD, Krutzik NJ. Extension of a model that aptly describes fracture of plain concrete to the impact analysis of reinforced concrete. In: Heki Shibata editor.. International Association for Structural Mechanics in Reactor Technology. Tokyo, Japan; 1991. p. 1-19.

[31] Slepyan LI. Crack in a material-bond lattice. J Mech Phys Solids 2005;53(6):1295-313.

[32] Schnaid F, Spinelli L, Iturrioz I, Rocha M. Fracture mechanics in ground improvement design. Ground Improv 2004;8:7-15.

[33] Sigmund O. Materials with prescribed constitutive parameters: an inverse homogenization problem. Int J Solids Struct 1994;31(17):2313-29.

[34] Silling SA. Reformulation of elasticity theory for discontinuities and long-range forces. J Mech Phys Solids 2000;48(1):175-209.

[35] Song J-H, Wang H, Belytschko T. A comparative study on finite element methods for dynamic fracture. Comput Mech 2007;42(2):239-50. doi:10.1007/s00466007-0210-X.

[36] Timoshenko SP, Young DH. Vibration problems in engineering. 3rd ed. Princeton, New Jersey: D. Van Nostrand Company; 1955.

[37] Xu X-P, Needleman A. Numerical simulation of fast crack growth in brittle solids. J Mech Phys Solids 1994;42(9):1397-434. 